\title{
Comments
}

\section{Comments on "A Representation for the Symbol Error Rate Using Completely Monotone Functions"}

Berkan Dulek, Member, IEEE

\begin{abstract}
It was shown in the above-titled paper by Rajan and Tepedelenlioglu (see ibid., vol. 59, no. 6, p. 3922-31, June 2013) that the symbol error rate (SER) of an arbitrary multidimensional constellation subject to additive white Gaussian noise is characterized as the product of a completely monotone function with a nonnegative power of signal-to-noise ratio (SNR) under minimum distance detection. In this comment, it is proved that the probability of correct decision of an arbitrary constellation admits a similar representation as well. Based on this fact, it is shown that the stochastic ordering $\left\{\leq \mathcal{G}_{\alpha}, \alpha \geq 0\right\}$ proposed by the authors as an extension of the existing Laplace transform order to compare the average SERs over two different fading channels actually predicts that the average SERs are equal for any constellation of dimensionality smaller than or equal to $2 \alpha$. Furthermore, it is noted that there are no positive random variables $X_{1}$ and $X_{2}$ such that the proposed stochastic ordering is satisfied in the strict sense, i.e., $X_{1}<\mathcal{G}_{\alpha} X_{2}$, when $\alpha=N / 2$ for any positive integer $N$. Additional remarks are noted about the fading scenarios at low SNR and the generalization to additive compound Gaussian noise originally discussed in the subject paper.
\end{abstract}

Index Terms - Canonical representation, completely monotone, Gaussian noise, stochastic ordering, symbol error rate (SER).

\section{SYSTEM MODEL}

Following [1], we consider the standard baseband discrete-time system model for $M$-ary communications through AWGN, which is described as

$$
\mathbf{y}=\mathbf{s}+\mathbf{z},
$$

where the transmitted symbol $\mathbf{s} \in \mathbb{R}^{N}$ is drawn from a constellation $\mathcal{S}:=\left\{\mathbf{s}_{1}, \ldots, \mathbf{s}_{M}\right\}$. The noise is assumed to be a zero-mean multivariate Gaussian random vector with independent and identically distributed (i.i.d.) components, i.e., $\mathbf{z} \sim \mathcal{N}\left(\mathbf{0}, \sigma_{0}^{2} \mathbf{I}\right)$, where $\sigma_{0}^{2}$ is the noise variance per dimension and $\mathbf{I}$ denotes the identity matrix. More explicitly, the probability density function (PDF) of the noise $\mathbf{z}$ is

$$
f_{\mathbf{z}}(\mathbf{x})=\left(\frac{1}{2 \pi \sigma_{0}^{2}}\right)^{\frac{N}{2}} e^{-\frac{|\mathbf{x}|^{2}}{2 \sigma_{0}^{2}}}
$$

where $|\mathbf{x}|$ denotes the $\ell^{2}$ norm of $\mathbf{x}$, i.e., $|\mathbf{x}|=\sqrt{\mathbf{x}^{T} \mathbf{x}}$, and the superscript $(\cdot)^{T}$ denotes the transpose. Assuming that the average signal energy is normalized as $M^{-1} \sum_{i=1}^{M}\left|\mathbf{s}_{i}\right|^{2}=1$, the average $\mathrm{SNR}$ is defined as $\rho:=1 / \sigma_{0}^{2}$. At the receiver, the maximum likelihood (ML) detector is considered. Since the ML detector under

Manuscript received June 3, 2013; revised September 11, 2013; accepted November 3, 2013. Date of publication November 12, 2013; date of current version January 15, 2014.

The author is with the Department of Electrical and Electronics Engineering, Bilkent University, Ankara 06800, Turkey (e-mail: berkandulek@gmail.com). Communicated by R. F. H. Fischer, Associate Editor for Communications. Digital Object Identifier 10.1109/TIT.2013.2290712
AWGN is the minimum distance one [2], the detected symbol $\hat{\mathrm{s}}$ is given by

$$
\hat{\mathrm{s}}=\underset{\mathbf{s} \in \mathcal{S}}{\arg \min }|\mathbf{y}-\mathbf{s}| .
$$

Assuming that the origin is shifted to the constellation point $\mathbf{s}_{i}$, the decision region (Voronoi region) of $\mathbf{s}_{i}$, denoted by $\Omega_{i}$, is given by

$$
\Omega_{i}=\left\{\mathbf{x} \in \mathbb{R}^{N} \mid \mathbf{a}_{i, j}^{T} \mathbf{x} \leq b_{i, j}, j=1, \ldots, i-1, i+1, \ldots, M\right\},
$$

where $\mathbf{a}_{i, j}=\left(\mathbf{s}_{j}-\mathbf{s}_{i}\right) /\left|\mathbf{s}_{j}-\mathbf{s}_{i}\right|$, and $b_{i, j}=\left|\mathbf{s}_{j}-\mathbf{s}_{i}\right| / 2$. Since an intersection of $M-1$ half-spaces is defined in (4), $\Omega_{i}$ is a convex set [3]. It is noted that the decision region does not depend on $\rho$. Furthermore, the minimum distance of the constellation is given by $d_{\text {min }}=\min _{\mathbf{s}_{i}, \mathbf{s}_{j} \in \mathcal{S}, \mathbf{s}_{i} \neq \mathbf{s}_{j}}\left|\mathbf{s}_{i}-\mathbf{s}_{j}\right|$

The probability of correct decision $P_{c, i}(\rho)$, given that $\mathbf{s}=\mathbf{s}_{i}$ is transmitted, is given by [2]

$$
P_{c, i}(\rho)=\left(\frac{\rho}{2 \pi}\right)^{N / 2} \int_{\Omega_{i}} e^{-\frac{\rho|\mathbf{x}|^{2}}{2}} \mathrm{~d} \mathbf{x}
$$

Likewise, the probability of symbol error (also known as symbol error rate, SER) $P_{e, i}(\rho)$, given that $\mathbf{s}=\mathbf{s}_{i}$ is transmitted, is obtained by

$$
P_{e, i}(\rho)=\left(\frac{\rho}{2 \pi}\right)^{N / 2} \int_{\mathbb{R}^{N} \backslash \Omega_{i}} e^{-\frac{\rho|\mathbf{x}|^{2}}{2}} \mathrm{~d} \mathbf{x},
$$

where $\mathbb{R}^{N} \backslash \Omega_{i}=\left\{\mathbf{x} \in \mathbb{R}^{N} \mid \mathbf{x} \notin \Omega_{i}\right\}$. Clearly, $P_{e, i}(\rho)=1-P_{c, i}(\rho)$. The probability of error averaged over all constellation points is

$$
P_{e}(\rho)=\sum_{i=1}^{M} \operatorname{Pr}\left[\mathbf{s}=\mathbf{s}_{i}\right] P_{e, i}(\rho)=1-P_{c}(\rho),
$$

where $\operatorname{Pr}\left[\mathbf{s}=\mathbf{s}_{i}\right]$ denotes the a priori probability of transmitting $\mathbf{s}_{i}$.

Next, an additional remark about the dimension of the constellation is noted. Let the $N$-by- $M$ constellation matrix corresponding to $\mathcal{S}$ be defined as $\mathbf{S}:=\left[\mathbf{s}_{1}, \ldots, \mathbf{s}_{M}\right]$, and the rank of $\mathbf{S}$ be denoted by $N^{*}$. It is shown in [1] that for any $M$-ary signal constellation $\mathcal{S}$ in $\mathbb{R}^{N}$, an equivalent $M$-ary signal constellation $\mathcal{S}^{*}$ can be constructed in $\mathbb{R}^{N^{*}}$ with the same SER as that of $\mathcal{S}$. Therefore, without loss of generality, we work with the reduced constellation $\mathcal{S}^{*}$, whose reduced dimension is denoted with $N^{*}$.

\section{MAIN RESUlts}

In [1], it is shown that the SER of an arbitrary multidimensional constellation impaired by AWGN is characterized as the product of a completely monotone (c.m.) function with a nonnegative power of the signal-to-noise ratio (SNR) under minimum distance detection. (See [1] and the references therein for information about c.m. functions.) The corresponding theorem in [1] is given below with a correction for the support of the representing function. More precisely, a factor of 0.5 is forgotten while substituting $u=r^{2} / 2$ into the argument of the indicator function in [1, Eq. (25)].

\footnotetext{
${ }^{1}$ Recalling that matched filtering and sampling would eliminate any unnecessary dimensions in the discrete-time baseband model given in (1), the concept of reduced dimensionality may seem superfluous. However, we keep this notation to maintain consistency with the subject paper [1].
} 
Proposition 1 [1, Thm. 1]: For a constellation $\mathcal{S} \subseteq \mathbb{R}^{N}$, whose reduced constellation is $\mathcal{S}^{*}$ and reduced dimension is $N^{*} \geq 2$, the SER of the minimum distance detector under AWGN admits the representation

$$
P_{e}(\rho)=\rho^{\alpha} f_{c m}(\rho),
$$

where $f_{c m}(\rho)$ is c.m. and $\alpha \geq N^{*} / 2-1$. In (8), the representing function of $f_{c m}(\rho)$ satisfies $\mu(u)=0$ when $u<d_{\text {min }}^{2} / 8$, and $\mu(u) \geq 0$ otherwise, where $d_{\min }$ is the minimum distance of the constellation.

In the following, we first show that a similar representation also characterizes the probability of correct decision.

Proposition 2: For a constellation $\mathcal{S} \subseteq \mathbb{R}^{N}$, whose reduced constellation is $\mathcal{S}^{*}$ and reduced dimension is $N^{*} \geq 1$, the probability of correct decision of the minimum distance detector under AWGN admits the representation

$$
P_{c}(\rho)=\rho^{\alpha} g_{c m}(\rho),
$$

where $g_{c m}(\rho)$ is c.m. and $\alpha \geq N^{*} / 2$.

Proof: First, the integral given in (5) is expressed in hyperspherical coordinates $(r, \boldsymbol{\theta})$, where $r:=|\mathbf{x}|$, and $\boldsymbol{\theta}:=\left[\theta_{1}, \ldots, \theta_{N^{*}-1}\right]$ are the angles such that [4]

$$
\begin{aligned}
x_{1} & =r \cos \theta_{1} \\
x_{2} & =r \cos \theta_{2} \sin \theta_{1} \\
\vdots & \\
x_{N^{*}-1} & =r \cos \theta_{N^{*}-1} \sin \theta_{N^{*}-2} \cdots \sin \theta_{1} \\
x_{N^{*}} & =r \sin \theta_{N^{*}-1} \sin \theta_{N^{*}-2} \cdots \sin \theta_{1},
\end{aligned}
$$

and the hyperspherical volume element is given by

$$
\mathrm{d}^{N^{*}} V=\left|\operatorname{det} \frac{\partial(\mathbf{x})}{\partial(r, \boldsymbol{\theta})}\right| \mathrm{d} r \mathrm{~d} \theta_{1} \cdots \mathrm{d} \theta_{N^{*-1}},
$$

where

$$
\left|\operatorname{det} \frac{\partial(\mathbf{x})}{\partial(r, \boldsymbol{\theta})}\right|=r^{N^{*}-1} \sin ^{N^{*}-2} \theta_{N^{*}-2} \sin ^{N^{*}-3} \theta_{N^{*}-3} \cdots \sin \theta_{1} .
$$

The range of the angles are

$$
0 \leq \theta_{l} \leq \pi, l=1, \ldots, N^{*}-2,0 \leq \theta_{N^{*}-1} \leq 2 \pi,
$$

which is denoted with $\boldsymbol{D} \boldsymbol{\theta}$. For simplicity, we also define

$$
f_{\boldsymbol{\theta}}(\boldsymbol{\theta}):=\frac{\Gamma\left(N^{*} / 2\right)}{2 \pi^{N^{*} / 2}} \sin ^{N^{*}-2} \theta_{N^{*}-2} \sin ^{N^{*}-3} \theta_{N^{*}-3} \cdots \sin \theta_{1},
$$

where $\Gamma(\cdot)$ is the Gamma function [4]. Note that $f_{\boldsymbol{\theta}}(\boldsymbol{\theta})$ can be regarded as a PDF over the range $D \boldsymbol{\theta}$ for $N^{*} \geq 2$. For $N^{*}=1$, we extend this definition by $f_{\theta}(\theta):=0.5(\delta(\theta)+\delta(\theta-\pi))$, where $\delta(\cdot)$ is the Dirac delta function. With these substitutions, the integral in (5) can be reformulated as

$$
P_{c, i}(\rho)=\frac{\rho^{N^{*} / 2}}{2^{N^{*} / 2-1} \Gamma\left(N^{*} / 2\right)} \int_{D \boldsymbol{\theta}} \int_{0}^{R_{i}(\boldsymbol{\theta})} e^{-\frac{\rho r^{2}}{2}} r^{N^{*}-1} f_{\boldsymbol{\theta}}(\boldsymbol{\theta}) \mathrm{d} r \mathrm{~d} \boldsymbol{\theta},
$$

where $R_{i}(\boldsymbol{\theta})$ is the distance between the origin and the boundary of the decision region $\Omega_{i}$ at the direction $\boldsymbol{\theta}$. Substituting $u=r^{2} / 2$ in (15), we get

$$
P_{c, i}(\rho)=\rho^{N^{*} / 2} \int_{D \boldsymbol{\theta}} \int_{0}^{R_{i}^{2}(\boldsymbol{\theta}) / 2} \frac{e^{-\rho u} u^{N^{*} / 2-1}}{\Gamma\left(N^{*} / 2\right)} f_{\boldsymbol{\theta}}(\boldsymbol{\theta}) \mathrm{d} u \mathrm{~d} \boldsymbol{\theta} .
$$

With the help of indicator function, ${ }^{2}$ the order of integration can be changed, and (16) can be expressed equivalently as

$$
P_{c, i}(\rho)=\rho^{N^{*} / 2} \int_{0}^{\infty} e^{-\rho u} \mu_{i}(u) \mathrm{d} u,
$$

where $\mu_{i}(u)$ is given by

$$
\mu_{i}(u)=\frac{u^{N^{*} / 2-1}}{\Gamma\left(N^{*} / 2\right)} \int_{D \boldsymbol{\theta}} \mathrm{I}\left[u \leq R_{i}^{2}(\boldsymbol{\theta}) / 2\right] f_{\boldsymbol{\theta}}(\boldsymbol{\theta}) \mathrm{d} \boldsymbol{\theta} .
$$

From (18), it is seen that $\mu_{i} \geq 0$ for $u \geq 0$. Then, the integral in (17) is a c.m. function of $\rho$ via Bernstein's theorem [1], [5], and $P_{c, i}(\rho)$ can be represented as $P_{c, i}(\rho)=\rho^{N^{*} / 2} g_{c m, i}(\rho)$, where $g_{c m, i}(\rho)$ is a c.m. function. From (7) and (17), we also get

$$
P_{c}(\rho)=\rho^{N^{*} / 2} \int_{0}^{\infty} e^{-\rho u} \mu(u) \mathrm{d} u,
$$

where $\mu(u):=\sum_{i=1}^{M} \operatorname{Pr}\left[\mathbf{s}=\mathbf{s}_{i}\right] \mu_{i}(u)$. Consequently, $P_{c}(\rho)=\rho^{N^{*} / 2} g_{c m}(\rho)$, where $g_{c m}(\rho)$ is a c.m. function via Bernstein's theorem since $\mu(u) \geq 0$ is assured. The generalization for $\alpha \geq N^{*} / 2$ in (9) follows by noting that for $k \geq 0, \rho^{-k} g_{c m}(\rho)$ is c.m whenever $g_{c m}(\rho)$ is c.m.

It should be noted that the representation given in (9) also applies to the more general case when the decision region of $\mathbf{s}_{i}$ corresponding to the minimum distance detector is replaced with an arbitrary measurable set $\Omega_{i} \subseteq \mathbb{R}^{N^{*}}$. To see this, let $\Omega_{i}^{\boldsymbol{\theta}}$ denote the set of points that belong to the decision region $\Omega_{i}$ at the direction $\theta$ (assuming that the origin is shifted to $\mathbf{s}_{i}$ ). Let $\mathrm{R}_{i}^{\boldsymbol{\theta}}$ be defined as the set of radial distances between the origin and the points that belong to the set $\Omega_{i}^{\boldsymbol{\theta}}$, i.e., $\mathrm{R}_{i}^{\boldsymbol{\theta}}:=\left\{|\mathbf{x}|: \mathbf{x} \in \Omega_{i}^{\boldsymbol{\theta}}\right\}$. Likewise, let $\mathrm{U}_{i}^{\boldsymbol{\theta}}$ be defined as $\cup_{i}^{\boldsymbol{\theta}}:=\left\{|\mathbf{x}|^{2} / 2: \mathbf{x} \in \Omega_{i}^{\boldsymbol{\theta}}\right\}$. Consequently, the inner integrals in (15) and (16) are computed over the points $r \in \mathrm{R}_{i}^{\boldsymbol{\theta}}$ and $u \in \mathrm{U}_{i}^{\boldsymbol{\theta}}$, respectively. Then, it can be seen that the representation in (9) is still valid by substituting $\mathrm{I}\left[u \in \mathrm{U}_{i}^{\boldsymbol{\theta}}\right]$ in place of $I\left[u \leq R_{i}^{2}(\boldsymbol{\theta}) / 2\right]$ in (18), which does not affect the nonnegativity of the resulting integral for $\mu_{i}(u)$.

Before proceeding further, an additional remark is noted. Based on (17) and (18), the correct decision probability of an arbitrary constellation (with reduced dimension $N^{*}$ ) subject to the AWGN and minimum distance detection can be alternatively expressed as

$$
P_{c}(\rho)=\sum_{i=1}^{M} \operatorname{Pr}\left[\mathbf{s}=\mathbf{s}_{i}\right] \mathbb{E}_{U, \boldsymbol{\theta}}\left\{\mathrm{I}\left[U \leq R_{i}^{2}(\boldsymbol{\theta}) / 2\right]\right\},
$$

where $U$ is a Gamma distributed random variable (RV) with shape parameter $N^{*} / 2$ and scale parameter $1 / \rho, \theta$ is distributed as given in (14), $U$ and $\boldsymbol{\theta}$ are independent, and $\mathbb{E}_{U, \boldsymbol{\theta}}\{\cdot\}$ denotes an expectation over their distributions. Likewise, we have $P_{e}(\rho)=\sum_{i=1}^{M} \operatorname{Pr}[\mathbf{s}=$ $\left.\mathbf{s}_{i}\right] \mathbb{E}_{U, \boldsymbol{\theta}}\left\{\mathrm{I}\left[U \geq R_{i}^{2}(\boldsymbol{\theta}) / 2\right]\right\}$.

In order to demonstrate the benefits of the representation given in Proposition 1 [1, Thm. 1], the authors defined a new stochastic ordering, which was then exploited to compare the average SER performance of an arbitrary multidimensional constellation over two different fading AWGN channels. The stochastic ordering $\leq \mathcal{G}_{\alpha}$ was defined as below.

Definition [1, Def. 2]: Let $X_{1}$ and $X_{2}$ be two positive RVs, and let $\alpha \geq 0$ be fixed. Then, $X_{1} \leq \mathcal{G}_{\alpha} X_{2}$ if and only if $\mathbb{E}\left\{X_{1}^{\alpha} e^{-\rho X_{1}}\right\} \geq$ $\mathbb{E}\left\{X_{2}^{\alpha} e^{-\rho X_{2}}\right\}$ for all $\rho>0$.

The authors also provided a necessary and sufficient condition.

\footnotetext{
${ }^{2}$ The indicator function is defined as $\mathrm{I}[x \in \mathrm{A}]=1$ if $x \in \mathrm{A}$, and 0 otherwise.
} 
Proposition 3 [1, Thm. 2]: Let $X_{1}$ and $X_{2}$ be two positive RVs, and $\alpha \geq 0$. Then, $X_{1} \leq \mathcal{G}_{\alpha} X_{2}$ if and only if

$$
\mathbb{E}\left\{X_{1}^{\alpha} f_{c m}\left(X_{1}\right)\right\} \geq \mathbb{E}\left\{X_{2}^{\alpha} f_{c m}\left(X_{2}\right)\right\},
$$

where $f_{c m}$ is c.m.

The stochastic ordering $\leq \mathcal{G}_{\alpha}$ was proposed as a generalization of the existing Laplace transform (LT) order [6], which corresponds to the case $\alpha=0$ in the above definition. Using LT ordering, generic comparisons of averages of a c.m. function over two different positive RVs can be obtained [6]. In an earlier paper [7, Thm. 1], the authors showed that the SER of arbitrary one- and two-dimensional constellations impaired by AWGN is c.m. under ML detection, and they successfully applied the LT ordering to compare the average SERs of commonly used parametric fading models such as Nakagami and Rician, which are monotonic in their line-of-sight parameters with respect to the LT order. In the subject paper [1, Corol. 1], a new derivation for the complete monotonicity of the SER of arbitrary one- and two-dimensional constellations was presented based on representation given in (8) along with a discussion regarding the application of the LT ordering. On the other hand, since the exponent of $\rho$ in (8) is positive for higher dimensional constellations $\left(N^{*} \geq 3\right)$, the LT ordering cannot be exploited. To facilitate generic comparisons of the average SER of arbitrary higher dimensional constellations over two different fading channels, the authors defined a new stochastic ordering $\leq \mathcal{G}_{\alpha}$ for $\alpha \geq 0$.

As an application of the proposed stochastic ordering, the authors considered an AWGN channel model under quasi-static fading. With perfect channel state information at the receiver, it is well-known that the average SER can be computed by integrating the instantaneous SER in AWGN over the distribution of the instantaneous channel power gain [8, Section 6.3.2]. More precisely, $\bar{P}_{e}(\rho)=\mathbb{E}\left\{P_{e}(\rho X)\right\}$, where $X$ denotes the instantaneous channel power gain and $\bar{P}_{e}$ is the average SER. Recalling that the instantaneous SER of an arbitrary constellation with reduced dimension $N^{*}$ admits the representation $P_{e}(\rho X)=\rho^{\alpha} X^{\alpha} f_{c m}(\rho X)$ for $\alpha \geq N^{*} / 2-1$, the authors applied the stochastic ordering relation to conclude that [1, Eq.(16)]

$$
X_{1} \leq \mathcal{G}_{\alpha} X_{2} \Rightarrow \mathbb{E}\left\{P_{e}\left(\rho X_{1}\right)\right\} \geq \mathbb{E}\left\{P_{e}\left(\rho X_{2}\right)\right\}, \forall \rho>0,
$$

where $X_{1}$ and $X_{2}$ represent the instantaneous channel power gains of two fading scenarios under AWGN. The above statement implies that if $X_{1} \leq \mathcal{G}_{\alpha} X_{2}$ for $\alpha=N^{*} / 2-1$, the average SER performance under $X_{2}$ is at least as good as that under $X_{1}$ over all average SNRs for all constellations with reduced dimension $N^{*}$. However, the stochastic ordering $\leq_{\mathcal{G}_{\alpha}}$ poses further implications, which raise concerns about its usefulness.

Proposition 4: Let $X_{1}$ and $X_{2}$ represent the instantaneous channel power gains of two fading scenarios under AWGN such that $X_{1} \leq \mathcal{G}_{\alpha}$ $X_{2}$ is satisfied for some positive $\alpha$. Then, the average SER under $X_{1}$ is identical to that under $X_{2}$ over all average SNRs for any constellation with reduced dimension $N \leq 2 \alpha$.

Proof: Consider an arbitrary constellation with reduced dimension $N$. Let $N / 2 \leq \alpha$, and $X_{1} \leq \mathcal{G}_{\alpha} \quad X_{2}$ is satisfied. Recall that $X_{1} \leq \mathcal{G}_{\alpha} \quad X_{2}$ implies $X_{1} \leq \mathcal{G}_{\beta} X_{2}$ for $0 \leq \beta \leq \alpha$ [1, Thm. 3]. Consequently, we have $X_{1} \leq \mathcal{G}_{N / 2} X_{2}$. Based on Proposition 1 [1, Thm. 1], the average SER under $X_{1}$ can be expressed as $\mathbb{E}\left\{P_{e}\left(\rho X_{1}\right)\right\}=\rho^{N / 2} \mathbb{E}\left\{X_{1}^{N / 2} f_{c m}\left(\rho X_{1}\right)\right\}$. Let $\tilde{f}_{c m}\left(X_{1}\right):=$ $f_{c m}\left(\rho X_{1}\right)$, which is also a c.m. function of $X_{1} \forall \rho>0$. Via Proposition $3\left[1\right.$, Thm. 2], we get $\rho^{N / 2} \mathbb{E}\left\{X_{1}^{N / 2} \tilde{f}_{c m}\left(X_{1}\right)\right\} \geq$ $\rho^{N / 2} \mathbb{E}\left\{X_{2}^{N / 2} \tilde{f}_{c m}\left(X_{2}\right)\right\}$. Substituting $\tilde{f}_{c m}\left(X_{2}\right)=f_{c m}\left(\rho X_{2}\right)$, it is concluded that $\mathbb{E}\left\{P_{e}\left(\rho X_{1}\right)\right\} \geq \mathbb{E}\left\{P_{e}\left(\rho X_{2}\right)\right\} \forall \rho>$ 0 . Now, consider the average probability of correct decision. From Proposition 2, $\mathbb{E}\left\{P_{c}\left(\rho X_{1}\right)\right\}=\rho^{N / 2} \mathbb{E}\left\{X_{1}^{N / 2} g_{c m}\left(\rho X_{1}\right)\right\}$.
Using Proposition 3 [1, Thm. 2] in conjunction with a similar argument, we get $\mathbb{E}\left\{P_{c}\left(\rho X_{1}\right)\right\} \geq \mathbb{E}\left\{P_{c}\left(\rho X_{2}\right)\right\} \forall \rho>0$, which in turn implies that $\mathbb{E}\left\{P_{e}\left(\rho X_{1}\right)\right\} \leq \mathbb{E}\left\{P_{e}\left(\rho X_{2}\right)\right\} \forall \rho>0$ since $P_{e}=$ $1-P_{c}$. Combining both results, it is concluded that if $X_{1} \leq \mathcal{G}_{\alpha} X_{2}$, $\mathbb{E}\left\{P_{e}\left(\rho X_{1}\right)\right\}=\mathbb{E}\left\{P_{e}\left(\rho X_{2}\right)\right\} \forall \rho>0$ for any constellation with reduced dimension $N \leq 2 \alpha$.

Although the stochastic ordering $\leq \mathcal{G}_{\alpha}$ was proposed in [1, Def. 2] as an extension of the LT to compare the average SERs of arbitrary higher dimensional constellations over different fading channels, it actually predicts that the average SER performances are equal at all average SNRs when the reduced dimension of the constellation satisfies $N^{*} \leq 2 \alpha$. To elucidate further, we consider the following example. Suppose that we would like to compare the average SERs of an arbitrary constellation with reduced dimension $N^{*} \geq 3$ over two fading channels denoted by $X_{1}$ and $X_{2}$ using the proposed stochastic ordering $\leq \mathcal{G}_{\alpha}$. From Proposition 1 [1, Thm. 1], it is seen that the smallest value for the exponent of $\rho$ in the representation for the SER given in (8) is $N^{*} / 2-1$. Therefore, we need the stochastic ordering $\leq \mathcal{G}_{\alpha}$ to hold for $\alpha=N^{*} / 2-1$ in order to conclude the result stated in (22). But, if $X_{1} \leq \mathcal{G}_{\alpha} X_{2}$ is satisfied for $\alpha=N^{*} / 2-1$, Proposition 4 implies that the average SER under $X_{1}$ is identical to that under $X_{2}$ over all average SNRs for any constellation with reduced dimension $N \leq 2 \alpha=N^{*}-2$. Although Proposition 4 does not provide any information about the comparison of the SER performances for an arbitrary constellation of dimensionality $N^{*}$, it does imply that the SER performances are identical for any constellation of dimensionality $N \leq N^{*}-2$ assuming that $X_{1} \leq \mathcal{G}_{N^{*} / 2-1} X_{2}$ is satisfied. This is a rather undesirable consequence for a stochastic ordering proposed to compare the average SERs of arbitrary higher dimensional constellations over different fading channels. On the other hand, for arbitrary one- and two-dimensional constellations, the LT ordering can be employed to satisfactorily serve this purpose due to its connection with c.m. functions as explored by the authors in [7].

The following proposition raises further concerns about the usefulness of the proposed stochastic ordering.

Proposition 5: There are no two positive RVs that satisfy $X_{1}<\mathcal{G}_{\alpha}$ $X_{2}$ when $\alpha=N / 2$ for any positive integer $N$.

Proof: Immediate from the proof of Proposition 4 by employing strict inequalities in [1, Def. 2] and [1, Thm. 2].

In the following, we present some additional remarks about the generalizations of Proposition 1 [1, Thm. 1] and its corollaries to the case of additive compound Gaussian noise, which were discussed in [1, Sec. IV.B]. In this case, the system model given in (1) is considered for $\mathbf{z}=\sqrt{W} \mathbf{g}$, where $W$ is a positive $\mathrm{RV}$, which is independent of $\mathbf{g}$, and $\mathbf{g}$ is an $N$-dimensional multivariate Gaussian with i.i.d. components, i.e., $\mathbf{g} \sim \mathcal{N}(\mathbf{0}, 1 / \rho \mathbf{I})$. The ML detector is still the minimum distance detector [9]. Furthermore, the SER of the reduced constellation is identical to the SER of the original one in this case as well [1].

The SER conditioned on $W=w$ is given by $P_{e}(\rho / w)$. Averaging over the distribution of $W$, the SER in the case of additive compound Gaussian noise can be computed as $\hat{P}_{e}(\rho)=\mathbb{E}\left\{P_{e}(\rho / W)\right\}$, where $(\hat{)})$ is used to distinguish the SER corresponding to the additive compound Gaussian noise from that corresponding to the conditional SER. For $N^{*} \leq 2, P_{e}(\rho)$ is known to be c.m. [7], and $P_{e}(\rho / w)$ directly inherits this property since $w$ is positive. Complete monotonicity of $\hat{P}_{e}(\rho)$ follows from the fact that a positive linear combination of c.m. functions is also c.m. For $N^{*}>2$, we assume that the PDF of RV $W$ has bounded support, i.e., $f_{W}(w)=0 \forall w \notin\left[w_{1}, w_{2}\right]$. This assumption is required to specify the support of the representing function $\mu(u)$ in the extension of [1, Thm. 1] and [1, Cor. 2] to the compound Gaussian noise case. Substituting (8) for $P_{e}(\rho)$, the following expression is obtained 
for $\hat{P}_{e}(\rho)$.

$$
\hat{P}_{e}(\rho)=\rho^{\alpha} \int_{0}^{\infty} e^{-\rho u} \hat{\mu}(u) \mathrm{d} u,
$$

where $\hat{\mu}(u)$ is given by

$$
\hat{\mu}(u)=\int_{w_{1}}^{w_{2}} \frac{\mu(u w)}{w^{\alpha-1}} f_{W}(w) \mathrm{d} w,
$$

where $\mu(u)$ is the representing function of $f_{c m}(\rho)$ given in (8). Since $w$ is positive and $\mu(u) \geq 0$ for $u \geq 0$, it follows that $\hat{\mu}(u) \geq 0$ for $u \geq 0$. Hence, $\hat{P}_{e}(\rho)$ admits the representation $\hat{P}_{e}(\rho)=\rho^{\alpha} \hat{f}_{c m}(\rho)$ where $\hat{f}_{c m}(\rho)$ is c.m. and $\alpha \geq N^{*} / 2-1$. However, it should be noted that the support of the representing function of $\hat{f}_{c m}(\rho)$ is now determined as follows: $\hat{\mu}(u)=0$ when $u<d_{\text {min }}^{2} /\left(8 w_{2}\right)$, and $\hat{\mu}(u) \geq 0$ otherwise. Likewise, the extension of the sufficient condition for the convexity of the SER can be correctly stated as follows.

Corollary [1, Extension of Corollary 2]: Consider a compound Gaussian noise channel determined by the PDF $f_{W}(w)$, which has bounded support $f_{W}(w)=0 \forall w \notin\left[w_{1}, w_{2}\right]$. If the reduced dimension $N^{*}$ of a constellation $\mathcal{S}$ is greater than two, the SER of the minimum distance detector under compound Gaussian noise satisfies $\hat{P}_{e}^{\prime \prime}(\rho) \geq 0$ when $\rho \geq \rho_{0}$, where $\rho_{0}:=8 w_{2}(\alpha+\sqrt{\alpha}) / d_{\text {min }}^{2}$, $\alpha=N^{*} / 2-1$, and $d_{\min }$ is the minimum distance of the constellation.

Contrary to the claim at the end of Sec. IV.B in [1], the sufficient condition given in the corollary requires the additional assumption of bounded support. It is also seen that the bound for convexity depends explicitly on the upper bound of the RV $W$.

Finally, we focus on the analysis preceding Theorem 3 in [1] about the performance of the AWGN channel being worse than a Nakagami$m$ channel in terms of the SER of constellations with $N^{*}>2$ at low SNR. The claim that $\mathbb{E}\left\{P_{e}\left(\rho X_{1}\right)\right\} \geq \mathbb{E}\left\{P_{e}\left(\rho X_{2}\right)\right\}$ for $\rho \leq \rho_{1}$ does not follow from the observation that $\left.\mathbb{E}\left\{X_{1}^{\alpha} e^{-\rho X_{1}}\right\} \geq \mathbb{E}\left\{X_{2}^{\alpha} e^{-\rho X_{2}}\right\}\right\}$ for $\rho \leq \rho_{1}$. To this end, observe that

$$
\mathbb{E}\left\{P_{e}\left(\rho X_{1}\right)\right\}=\rho^{\alpha} \int_{0}^{\infty} \mathbb{E}\left\{X_{1}^{\alpha} e^{-\rho u X_{1}}\right\} \mu(u) \mathrm{d} u .
$$

For the claim to hold, it is sufficient that $\rho \leq \rho_{1} / u$ for all $u$ in the support of $\mu(u)$, i.e., $u \geq d_{\min }^{2} / 8$. However, since $u$ can assume arbitrarily large values with $\mu(u)>0$, the upper bound $\rho_{1} / u$ gets very small. Hence, the claim on the low SNR scenario was not justified in the example given by the authors.

\section{REFERENCES}

[1] A. Rajan and C. Tepedelenlioglu, "A representation for the symbol error rate using completely monotone functions," IEEE Trans. Inf. Theory, vol. 59, no. 6, pp. 3922-3931, Jun. 2013.

[2] J. G. Proakis, Digital Communications, 4th ed. New York, NY, USA: McGraw-Hill, 2001.

[3] S. Boyd and L. Vandenberghe, Convex Optimization. Cambridge, U.K.: Cambridge Univ. Press, 2004.

[4] T. W. Anderson, An Introduction to Multivariate Statistical Analysis, 3rd ed. Cambridge, U.K.: Wiley, 2003.

[5] R. Schilling, R. Song, and Z. Vondraček, Bernstein Functions: Theory and Applications. Berlin, Germany: Walter de Gruyter, 2010.

[6] M. Shaked and J. G. Shanthikumar, Stochastic Orders and Their Applications, 1st ed. New York, NY, USA: Springer-Verlag, 1994.

[7] A. Rajan and C. Tepedelenlioglu, "On the complete monotonicity of symbol error rates," in Proc. IEEE ISIT, Jul. 2012, pp. 1430-1434.

[8] A. J. Goldsmith, Wireless Communications. New York, NY, USA: Cambridge Univ. Press, 2005.

[9] E. Conte, M. Di Bisceglie, M. Longo, and M. Lops, "Canonical detection in spherically invariant noise," IEEE Trans. Commun., vol. 43, nos. 2-4, pp. 347-353, Feb./Apr. 1995.

Berkan Dulek (S'11-M'13) received the B.S., M.S. and Ph.D. degrees in electrical and electronics engineering from Bilkent University in 2003, 2006 and 2012, respectively. From 2007 to 2010, he worked at Tubitak Bilgem Iltaren Research and Development Group. From 2012 to 2013, he was a postdoctoral research associate at the Department of Electrical Engineering and Computer Science, Syracuse University, Syracuse, NY. Currently, he is a postdoctoral research associate at the Department of Electrical and Electronics Engineering, Bilkent University, Ankara, Turkey. His research interests are in statistical signal processing, detection and estimation theory, and wireless communications. 